\title{
DISPOSICIONES PSICOLÓGICAS HACIA LA CORRUPCIÓN EN ESCOLARES Y UNIVERSITARIOS DE LA CIUDAD DE LIMA
}

\section{PSYCHOLOGICAL DISPOSITIONS TOWARDS THE CORRUPTION IN STUDENTS AND COLLEGE STUDENTS OF THE CITY OF LIMA}

\author{
LUIS VICUÑA PERI ${ }^{1}$, HECTOR HERNÁNDEZ VALZ, JOSÉ RIOS DIAZ, QUILICHE \\ VARGAS JUAN CARLOS, SANTILLANA PISCOYA CHRISTIAN, SARMIENTO \\ BARRENECHEA EDGAR, TIMOTEO JIMÉNEZ ANTONI, PALOMINO CUEVA DICK, NOE \\ RETAMOZO JASSIN, MUNGUIA CAMARENA MIGUEL, TORRES MALCA JENNY
}

Facultad de Psicología UNMSM

\section{RESUMEN}

OBJETIVO: conocer las disposiciones psicológicas, en términos del estilo atribucional explicativo hacia la corrupción en escolares y universitarios en función al sexo y la edad cronológica. DISEÑO: el estudio es descriptivo transversal comparativo. MATERIAL Y MÉTODOS: el conocimiento del estilo atribucional, es la resultante de la combinación de tres dimensiones: externalidad, inestabilidad y especificidad, fue mediante la aplicación del test ASQ-CIDE, de Peterson, adaptado hacia los actos corruptos, la validez fue por el método de análisis de contenido, y la confiabilidad por el método del test retest. El mencionado test se aplicó en forma colectiva a grupos no mayores de veinticinco por vez, a una muestra seleccionada por estratos, escolares del nivel secundario y universitario, con un margen de error del $0.05 \mathrm{y}$ un nivel de confianza del 0.95. RESULTADOS: la tendencia de la muestra estudiada es hacia la internalidad, inestabilidad y especificidad que corresponde al estilo explicativo optimista en un 73 por ciento, en tanto que el 27 por ciento muestra tendencia hacia el estilo explicativo pesimista hacia los actos corruptos. CONCLUSIONES: En la dimensión Externalidad Internalidad la edad cronológica plantea diferencias significativas, a menor edad mayor Externalidad. En la dimensión Estabilidad e Inestabilidad, también la edad cronológica plantea diferencias significativas donde a menor edad hay mayor Inestabilidad. En la dimensión Especificidad - Globalidad la edad cronológica no plantea diferencias significativas, el grupo en su conjunto tiende hacia la Especificidad. Finalmente, el sexo no plantea diferencias en las dimensiones mencionadas, por lo que resulta alentador que nuestros jóvenes se muestren optimistas para enfrentar a los actos de corrupción.

Palabras Claves: Estilo, Atribución, Optimista, Explicativo, Corrupción.

\begin{abstract}
OBJECTIVE: to know the dispositions psychological, in terms of the explanatory atribucional style towards the corruption in students and college students in function to sex and the chronological age. DESIGN: the study is descriptive cross-sectional comparative. MATERIAL And METHODS: the knowledge of the atribucional style, is the resultant of the combination of three dimensions: externalidad, instability and specificity, were by means of the application of test ASQ-CIDE, of Peterson, adapted towards the corrupt acts, the validity was by the method of content analysis, and the trustworthiness by the method
\end{abstract}

\footnotetext{
${ }^{1}$ Email: luisvicunaperi@hotmail.com luisvicunaperi@yahoo.com
} 
of the test retest. The mentioned test was applied in collective form to non greater groups of twenty-five per time, to a sample selected by layers, students of the secondary level and university, with a margin of error of a 0,05 and level of confidence of the 0.95. RESULTS: the tendency of the studied sample is towards the Internally, instability and specificity that corresponds to the optimistic explanatory style in a 73 percent, whereas the 27 percent shows tendency towards the pessimistic explanatory style towards the corrupt acts. CONCLUSIONS: In the Externally dimension Internally the chronological age raises significant differences, to smaller age greater Externalidad. In the dimension Stability Instability, also the chronological age raises significant differences where to smaller age there is greater Instability In the dimension Specificity - Totality the chronological age does not raise significant differences, the group as a whole tends towards the Specificity. Finally, sex does not raise differences in the mentioned dimensions, reason why it is encouraging that our young people are optimists to face the corruption acts.

Keywords: Style, Attribution, Optimist, Explanatory, Corruption.

\section{INTRODUCCIÓN}

Identificar las disposiciones psicológicas de los púberes, adolescentes escolares y jóvenes universitarios hacia la corrupción, nos permitirá conocer los sentimiento expresados de nuestra juventud hacia los mecanismos de control y disminución de la conducta corrupta, averiguando si el control viene del individuo o por lo contrario si es objeto de la presión y control externo, desde la teoría de Rotter (1966)1 y desde el enfoque de Peterson y Seligman (1991) 1 a qué atribuyen los comportamientos corruptos. Esto hace necesario revisar desde diferentes enfoques lo que se asume como corrupción dos puntos que desarrollaremos dentro del espacio permitido.

La revisión bibliográfica en nuestro medio, nos lleva a afirmar que aún este problema, no ha sido abordado, desde la perspectiva psicológica y lo más cercano a los estilos atribucionales y al locus de control (1) son los estudios acerca de las disposiciones psicológicas ante los diferentes tipos de afronte a estados de emergencia de origen social y natural, Vicuña $(1999)^{2}$ y en los sentimientos de concordancia entre los intereses vocacionales con los estilos atribucionales de las causas que determinaron el ingreso a la Universidad Nacional Mayor de San Marcos, Vicuña (2001) ${ }^{3}$. En el extranjero se ha realizado numerosas investigaciones acerca de la corrupción y también sobre los estilos atribucionales (2) y locus de control (1) pero no asociados con el comportamiento corrupto, como daremos cuenta más adelante.

El Estilo Atribucional, en los avances de la Psicología sobre el control personal, según Peterson \& Stunkard $(1991)^{1}$, el "Estilo Explicativo», es un constructo mas comprehensivo y con mayor pode explicativo, que el de Locus de Control formulado por $\mathbf{J}$. B. Rotter, ${ }^{1}$ y el de Autoeficacia de A. Bandura (1977) ${ }^{4}$. Aunque no los sustituya totalmente, comparte con estas y otras teorías del control personal la: siguientes características.

- El control personal es uno de los modos importantes en que las personas se diferencian unas de otras.

- El control personal reside en la transacción entre la persona y el mundo; no es sólo una disposición o sólo una característica de ambiente.

- El control personal puede tomar la forma de la creencia en que une puede afectar 
los resultados reales, elegir entre ellos, afrontar sus consecuencias, y/o entenderlas.

- En un ambiente retador, el control personal es deseable; este da ánimo a las emociones, la conducta y el sistema fisiológico ante los retos.

- El control personal puede ser frustrado por el fracaso y animado por el éxito, aunque esta no sea una relación uno a uno con los patrones de éxitos y fracasos del pasado.

Bernard Weiner y Albert Stunkard, en el artículo publicado en $1992^{4}$, comparan las tres propuestas más conocidas que sobre Control Personal, se han venido desarrollando: Locus de control, autoeficacia y estilo explicativo. Todas ellas tienen en común su impacto en las conductas futuras de las personas, aunque en diferentes niveles.

Cada una de las teorías mencionadas se ocupa de diferentes dimensiones, a diversos niveles de abstracción o generalización, siendo el locus de control un factor más específico, mientras que el estilo explicativo llega a ser de tal nivel de generalidad que incluso puede postularse como un rasgo constitutivo de la personalidad.

Antes de revisar directamente los componentes del estilo explicativo o atribucional, veamos cómo se derivó ésta de la teoría atribucional de la motivación.

Bernard Weiner ${ }^{4}$ es uno de los psicólogos que ha procurado integrar los hallazgos en esta área de investigación formulando una teoría atribucional de la motivación para el éxito o motivación de logro (Weiner, 1992) ${ }^{4}$. Para él, las personas suelen atribuir sus éxitos o fracasos a una de las siguientes cuatro posibles causas: habilidad, cantidad de esfuerzo, dificultad de la tarea y la suerte.

\begin{tabular}{|l|c|c|}
\hline & Interno & Externo \\
\hline Estable & Habilidad & Dificultad \\
\hline Inestable & Esfuerzo & Suerte \\
\hline
\end{tabular}

Estas cuatro fuentes de causalidad se integran en dos dimensiones: Estable Inestable, e Interno - Externo. La habilidad es entonces una causa interna al sujeto y relativamente estable en el tiempo, la cantidad de esfuerzo es el otro elemento de la dimensión interna pero que puede fácilmente variar de una situación a otra, es decir no es estable en el tiempo. Mientras que la dificultad de la tarea es un aspecto externo al sujeto y relativamente estable mientras que el factor suerte es externo y no constante o inestable pues no está bajo control de la persona y puede variar de una ocasión a otra.

En diversas publicaciones Weiner4 presenta resultados de investigaciones que evidencian la influencia de las atribuciones de causalidad en la persistencia, intensidad y la elección de determinadas conductas (Weiner, 1992) ${ }^{4}$.

A diferencia de la teoría de la expectativa por valor de Atkinson, en la teoría atribucional de Weiner ${ }^{4}$ las reacciones emocionales y las expectativas de éxito o fracaso son mediadas por la atribución hecha sobre la situación percibidas antes de que el sujeto llegue a la acción (Weiner, 1992) ${ }^{4}$.

En el marco de la teoría atribucional de la motivación propuesta por Weiner ${ }^{4}$, y tomando como punto de partida el modelo reformulado de la desesperanza aprendida, Peterson ${ }^{1}$ incluye además de las dos dimensiones de atribución consideradas por Weiner4, 
una tercera que considera lo global o especifico del factor causal.

En el constructo denominado estilo explicativo o atribucional confluyen por un lado la teoría de la atribución y por otro, el de la desesperanza aprendida (Seligman, 1975) ${ }^{5}$, como un modo de explicar las diferencias individuales en las respuestas a eventos no controlables.

A partir de los estudios publicados por Seligman en 1975 y Abramson en $1978^{5}$, Peterson $(1991)^{1}$ identifica que el efecto de experiencias incontrolables y negativas producían en los sujetos la desesperanza aprendida pero en diversos modos. Por ello no se encontraban los mismos patrones que en los experimentos con animales. Tras varios años de investigación y una serie de formulaciones Peterson ${ }^{1}$ finalmente llegó a proponer como constitutivas del Estilo Explicativo las tres dimensiones causal es mencionadas: Interno Externo, Estable - Inestable y Global Específico.

El Estilo Explicativo es una variable cognitiva de personalidad que refleja el modo en que las personas explican habitualmente las causas de sus fracasos, da cuenta de la falta de motivación o desmoralización ante situaciones que demandan logros (Peterson, 1991) ${ }^{1}$.

Si una persona tiende a atribuir las razones de sus fracasos o eventos negativos a causas internas, entonces estará minando su propia autoestima, sentido de valía personal y percepción de su capacidad para controlar las situaciones.

Si a ello se aúna la tendencia a pensar que las causas de los infortunios sufridos van a permanecer más allá del día de hoy y de la situación específica presente, tendremos que la persona estará atribuyendo su condición a factores estables y globales.

La conjunción de una explicación interna, estable y global, es la que mayor pesimismo y desmoralización producirá en el individuo y es denominada por ello estilo explicativo pesimista.

Se ha encontrado que el estilo explicativo pesimista está asociado a la depresión e incluso a la pérdida de la salud físico (Peterson, 1991, 450) ${ }^{1}$, pero además de ellos Peterson ${ }^{1}$ cita los siguientes resultados como consecuentes de un estilo explicativo pesimista:

- Depresión (Sweeney, Anderson, \& Bailey, 1986) ${ }^{1}$

- Bajas calificaciones en la escuela (Peterson \& Barret, 1987) ${ }^{1}$

- Bajo desempeño laboral (Seligman \& Schulman, 1986) ${ }^{1}$

- Soledad (Anderson, Horowitz, \& French, 1983)1

- Mal estado de salud física (Peterson, Seligman \& Vaillant, 1988) ${ }^{1}$

De lo descrito se observa que el estilo atribucional, actúa como un rasgo de la personalidad mediante la cual ante los diferentes acontecimientos de la vida adoptamos posturas optimista o pesimista cuyas consecuencias de manera inmediata repercute sobre la buena salud en un lado y en el opuesto, trastornos psicosomáticos, los que tienen implicancias directas sobre el entorno social del individuo a mediano y largo plazo. Nuestro segundo componente de revisión es la corrupción, al respecto hay varios enfoques los que revisaremos brevemente.

Las definiciones de corrupción centradas en la conducta suelen sostener que la 
corrupción es el abuso de un cargo, del poder y de los recursos públicos para la obtención de beneficios personales. Johnston, 1993, en García y otros, 1999) ${ }^{6}$, es en cierta forma la opinión de una época dentro de un contexto dado las que determinan que un acto cualquiera, que «implica el perjuicio de un beneficio público para satisfacer uno privado, será considerado corrupto» (Johnston, 1993, citado en García y otros, 1999).

Otro enfoque para definir corrupción el de Heidenheimer (1989, en García y otros, $1999)^{6}$, quien ha diferenciado matices corrupción según las percepciones éticas de la clase política y la opinión pública. Se llama «corrupción negra» a aquellas acciones más potentes y universalmente aceptadas como el soborno y la extorsión. La llamada corrupción blanca ocurre en el caso en que actos corruptos son aceptados ampliamente por las personas en el ámbito que se trate. Entre la corrupción negra y blanca, se encuentra la corrupción gris, en la que se incluyen todas las conductas acerca de las cuales las elites y la opinión pública discrepan al momento de evaluarlas como corruptas o no.

Otra forma de entender el fenómeno de la corrupción es considerarlo tanto como un recurso y como un proceder. En la primera visión, la corrupción referida como un recurso expeditivo, la corrupción se utiliza para acelerar los procesos y, por tanto, el corrupto es «un experto en atajos». Esta característica racional por excelencia sustrae al corrupto del orden de la inadvertencia, siendo jamás inocente, pues siempre sabe lo que hace, está sujeto a la vigilia cognitiva, al darse cuenta. Desde la segunda perspectiva, la corrupción es un proceder, un modo de hacer para obtener beneficios. Este proceder tiene una característica sustancial, que es la desviación respecto de una cierta normatividad, o sea, la obtención del beneficio, se lleva a cabo, al margen de la conducta normal. La desviación puede hacerse respecto a una norma jurídica o normas éticas (Silva y Hernández, 1995) ${ }^{7}$

De las definiciones se desprenden las siguientes características del acto corrupto:

1. Toda acción corrupta, consiste en la trasgresión de una norma.

2. Se realiza para la obtención de un beneficio privado.

3. Surge dentro del ejercicio de una función asignada.

4. El individuo corrupto intenta siempre encubrir activamente su comportamiento.

Una definición integrativa de las características de corrupción es la entregada por Fernández - Dols (Fernandez-Dols, 1993) ${ }^{8}$ quien entiende la corrupción como una manipulación o transgresión encubierta de las normas que rigen una organización racional, con vistas a lograr, un beneficio privado.

Según Cartier-Bresson (1996, en Carda y otros, 1999) ${ }^{6}$, la corrupción puede ser caracterizada por la fórmula:

Corrupción $=$ Monopolio + discrecionalidad - transparencia .

La esencia más lesiva de la corrupción, radica en que importantes decisiones son determinadas por móviles ajenos al beneficio colectivo, sin importar las consecuencias que acarrean para la sociedad.

Las distintas acciones que se pueden clasificar como actos corruptos pueden ser clasificadas en 16 categorías, las cuales a su vez, pueden ser agrupadas en 3 grupos que se detallan a continuación 


\begin{tabular}{|c|c|}
\hline $\begin{array}{l}\text { l. Corrupción directa } \\
\text { Es aquella corrupción en que ocurre un } \\
\text { aprovechamiento directo de su función, } \\
\text { por parte del funcionario público, el } \\
\text { privado o el individuo particular, para } \\
\text { obtener un beneficio. }\end{array}$ & $\begin{array}{c}\text { Abuso de poder } \\
\text { Apropiación de bienes privados o públicos } \\
\text { El enriquecimiento ilícito } \\
\text { La extorsión } \\
\text { El favoritismo y el nepotismo }\end{array}$ \\
\hline $\begin{array}{l}\text { 2. Corrupción mediada } \\
\text { En estas acciones el beneficio particular } \\
\text { proviene de un tercero. }\end{array}$ & $\begin{array}{c}\text { La aceptación de ventajas indebidas El tráfico de } \\
\text { influencia } \\
\text { El soborno } \\
\text { El clientismo político } \\
\text { Las malas prácticas electorales }\end{array}$ \\
\hline $\begin{array}{l}\text { 3. Aprovechamiento de procedimientos } \\
\text { En este grupo de categorías el acto } \\
\text { corrupto consistiría en el aprovechamiento } \\
\text { de falencias en el sistema de } \\
\text { procedimientos en que se halla inserto el } \\
\text { individuo. }\end{array}$ & $\begin{array}{c}\text { El manejo indebido de bienes o fondos públicos } \\
\text { y/o privados. } \\
\text { El manejo indebido y el ocultamiento de } \\
\text { información. } \\
\text { La negociación incompatible Los manejos } \\
\text { indebidos de los procedimientos públicos. } \\
\text { El lavado de dinero. } \\
\text { Nepotismo. }\end{array}$ \\
\hline
\end{tabular}

Identificado los tipos de corrupción es necesario revisar algunos Modelos explicatorios de la corrupción:

\section{Modelo de la responsabilidad individual}

\section{a. De índole psicodinámica}

La corrupción es motivada por la necesidad individual del humano de poseer y controlar cada vez más, así en los grandes manejos de la corrupción lo que opera es una negación de la vulnerabilidad. (Huerta, 1992)

Para Anderson y Aresti (en Huerta, 1992)9, el corrupto es alguien que se miente, pero no se da cuenta de ello, porque en el fondo no quiere saber la verdad, «su verdad». Llegar a saberla, implica un dolor muchas veces intolerable, pues tendría que enfrentar el mundo de otro modo como está acostumbrado.

Un enfoque más personal, psicoanalítico, estima que las personas que comúnmente actúan de forma corrupta, creando grandes desfalcos, desde su infancia que para tener algo parecido al amor, tenían que comprarlo.

\section{b. Modelo filosófico-moral}

La sociedad, se expone a un desarrollo incontrolable y desaforado, alterando la estructura natural, sin que este crecimiento tome en consideración límite alguno. Esto parece ser uno de los signos característicos de nuestro tiempo, al que llaman «postmoderno».

Junto a este desarrollo de los procesos, asistimos a una suerte de retirada de la participación, resultando indiferente aquello que no depende directamente de uno. Este relajamiento de los valores colectivos, vindica sin miramientos la figura del individuo, produciéndose una exacerbación del ser para sí (Silva y Hernández, 1995) ${ }^{7}$.

Hoy día las personas se inclinan más por el pequeño mundo, que por mundo 
maximales. Hoy día, lo que seduce no es el bien común, sino excención, la prerrogativa individual (Foucault, en Silva y Hernández, 1995) ${ }^{7}$.

La sociedad postmoralista, se enmarca en la postmoderna y se caracteriza porque no hay obligación ni sanción. Hay un apego a los beneficios de los valores individualistas en que no resulta inmoral pensar en sólo uno mismo.

Además, hay un cambio en los valores del trabajo. Trabajar hoy día, es una actividad que en lo posible se debe evitar; se ha desvinculado de la obligación moral respecto a la colectividad, de tal modo que el trabajo para a ser una actividad esencialmente al servicio del individuo. Más aún, en lugar de la moral del civismo, tenemos el culto a la esfera privada y la indiferencia hacia la cosa pública, el afán por el dinero todopoderoso y la democratización de Ia corrupción. (Lipovetsky, en Silva y Hernández,1995) ${ }^{7}$.

\section{c. Modelos Sociológicos}

\section{Corrupción y burocracia}

Godoy (1996, en García y otros, 1999)6 plantea que existen diversos factores que contribuyen a la génesis de la corrupción, como son: los bajos ingresos de los agentes públicos, la ausencia de supervisión y de formación profesional, la falla en procedimientos contables que impiden detectar apropiaciones indebidas y el incremento de la burocracia gubernamental. Es importante aclarar que no es la burocracia en sí la causa de posibles actos corruptos, sino que en los países en vías de modernización la corrupción tiende a debilitar o perpetuar la debilidad de la burocracia gubernamental.

Desde una perspectiva institucional, por otra parte, la posibilidad de que se produzcan actos corruptos se incrementará si el marco institucional o las normas establecidas por un gobierno no establecen los incentivos y los medios de cumplimiento obligatorios adecuados para evitar que los funcionarios públicos y lo usuarios perciban mayores ventajas en infringir las normas que en cumplirlas (Mosqueira, 1995, en García y otros, 1999) $)^{6}$.

\section{Modelo económico-racional de la corrupción.}

Las explicaciones de elección racional suponen que el mecanismo de la corrupción es, en casi todo, semejante al que regula el mercado. Esto es, que los factores que se enfrentan a la posibilidad de violar las leyes hacen un cálculo de costo-beneficio, y que la corrupción prevalecerá siempre que sea rentable (Huntington y Scott, 1994)10.

Una autoridad corrupta ve a su cargo como un negocio cuyo ingreso intentará maximizar. El cargo se convierte, por lo tanto, en una unidad «maximizadora». La dimensión de sus ingresos depende de la situación del mercado y su talento para encontrar el punto de ganancia máxima en la curva de la demanda pública Johnston, 1968, en García y otros, 1999) $)^{6}$.

\section{d. Modelo Psicosocial de la «Norma perversa»}

Tanto para la visión clásica de la psicología social como para la actual el concepto de norma da por supuesto que los grupos producen sus propias normas, que éstas poseen una cierta funcionalidad y que el individuo puede adaptarse con mayor o menor éxito a dichas referencias (Fernández-Dols, 1993, p.92)8; la psicología social europea, por su parte, 
señala que las normas creadas por un grupo mayoritario pueden servir para discriminar y sojuzgar a los miembros de un grupo minoritario.

Según la conceptualización de Fernández-Dols ${ }^{8}$, las normas sociales no necesariamente son formadas por el mismo grupo $\mathrm{y}$, aunque este fuese el caso, no poseen siempre una clara funcionalidad.

Conceptual izaremos a la norma perversa como la «norma explícita e incumplible que un grupo asume o sufre por iniciativa de otro grupo o por propia iniciativa» (FernándezDols, 1993, p.95) ${ }^{8}$.

Por explícita se refiere al hecho de que un grupo de jueces ajenos a la norma podría predecir su cumplimiento con gran acuerdo, a partir del sentido común y del nivel de información que poseen.

Por incumplible se entiende que el cumplimiento de la norma se da en situaciones excepcionales o ideales.

Existen variadas condiciones que pueden llevar a que una norma devenga perversa. Entre ellas, podemos destacar:

- Ambientes rápidamente cambiantes y complejos, en los cuales las fuentes normativas antes respetadas son sistemáticamente desvalorizadas.

- Errores en la formulación y diseño de las normas, lo que las hace desde el primer momento inútiles. Estaría relacionado con la incompetencia de los legisladores.

- Mayor preocupación de la legitimidad que del cumplimiento de la norma, entendido esto como la preocupación excesiva porque el objetivo de la norma sea valioso, sin preocuparse de las posibilidades reales de cumplimiento ni de las dificultades inherentes a éste. Sociedad de validez/legitimidad. Se refiere a la preocupación principal que tendrían los poderes legisladores en la promulgación y aplicación de las normas, centrándose en las posibilidades prácticas de realización de la norma o en las consecuencias positivas finales de su cumplimiento, respectivamente. Este último enfoque es el de mayor riesgo, porque tiende a generar con mayor facilidad normas perversas; las culturas latinas tenderían a pertenecer a este grupo.

Las normas no se originan perversas, devienen perversas con el paso del tiempo. Se verifica su perversidad, primero, con el incumplimiento masivo. Pero esta no es condición suficiente: es necesario, además, que se produzcan efectos negativos en su incumplimiento para que se considere perversa.

Consecuencias de la norma perversa; las dos principales consecuencias de las normas perversas son la desmoralización y la corrupción. La desmoralización consistiría en la visión negativa de la autoridad encargada de imponer este tipo de normas y una mayor tolerancia a otro tipo de transgresiones.

La corrupción se traduciría, por una parte, en la aparición de fuentes de poder alternativas, capaces de manipular la situación a favor del individuo que trasgrede la norma e imponer otra norma alternativa en propio beneficio y, por otra parte, a una mayor tolerancia de la opinión pública frente a este tipo de acciones ilícitas. 
Estadísticas de la corrupción, actualmente con indicadores que se aproximan a ser confiables según Transparencia Internacional (T1) ${ }^{11}$ es una organización no gubernamental podemos conocer la posición de los países en función a los indicadores de corrupción. Esta organización entrega cada año el más completo índice de percepción de la corrupción en el mundo.

El índice de Percepción de la Corrupción (IPdC) es una «encuesta de encuestas», basada en numerosas encuestas con expertos y público en general acerca de sus puntos de vista sobre la extensión de la corrupción en muchos países alrededor del mundo, y busca ser un incentivo para los gobiernos de los distintos países para que luchen contra la corrupción.

El IPdC de Transparencia Internacional correspondiente a $1998^{11}$ cubre 85 países, siendo percibidos como los menos corruptos aquellos que alcanzan puntuaciones cercanas a 10.

A pesar de que el IPdC cubre un número récord de más de 80 países, son muchos los países que no han sido incluidos en el índice, debido a que no se dispone de suficientes datos confiables. El $\mathrm{IPdC}$ muestra que la corrupción no es percibida como una plaga exclusiva de los países en desarrollo. Muchos en Europa Central y Oriental y algunos de Latinoamérica tienen puntajes muy bajos y un número de países industrial izados tienen puntajes que revelan el grave problema que es la corrupción en estos países.

Desde su concepción, el IPdC ha servido al propósito constructivo de estimular el debate público acerca de la corrupción. En algunos países ha contribuido además a una sustantiva reforma anti-corrupción. El IPdC anual sensibiliza a la opinión pública en todo el mundo sobre la cuestión de la corrupción y constituye un factor de peso en las decisiones de inversión en el extranjero de las corporaciones multinacionales.

\section{Notas del IPdC de 1998}

El puntaje del IPdC de 1998 - se refiere a las percepciones del grado de corrupción, como es visto por los empresarios, analistas de riesgo y público en general, en una escala entre 10 (sumamente íntegro) y 0 (sumamente corrupto).

Encuestas utilizadas - se refiere al número de encuestas que estiman el desempeño de un país. Se utilizaron 12 encuestas y se ha requerido que exista información sobre un país, en al menos tres de estas encuestas, para que el país sea incluido en el índice de 1998 (Transparency International, 1999). 


\begin{tabular}{|c|c|c|c|c|}
\hline Pusición del pais & & & & \\
\hline País & IPdC de 1998 puntaje & Desviación estándar & Encuestas utilizadas & \\
\hline 1 & Dinamarca & 10.0 & 0.7 & 9 \\
\hline 2 & Finlandia & 9.6 & 0.5 & 9 \\
\hline 3 & Suecia & 9.5 & 0.5 & 9 \\
\hline 4 & Nueva Zelanda & 9,4 & 0.7 & 8 \\
\hline 5 & Islandia & 9.3 & 0.9 & 6 \\
\hline 6 & Canadá & 9.2 & 0.5 & 9 \\
\hline 7 & Singapur & 9.1 & 1.0 & 10 \\
\hline 8 & Holanda & 9.0 & 0.7 & 9 \\
\hline 9 & Noruega & 9.0 & 0,7 & 9 \\
\hline 10 & Suiza & 8.9 & 0.6 & 10 \\
\hline 11 & Australia & 8.7 & 0.7 & 8 \\
\hline 12 & Luxemburgo & 8.7 & 0.9 & 7 \\
\hline 13 & Reino Unido & 8.7 & 0.5 & 10 \\
\hline 14 & Irlanda & 8.2 & 1.4 & 10 \\
\hline 15 & Alemania & 7.9 & 04 & 10 \\
\hline 16 & Hong Kong & 7.8 & 1.1 & 12 \\
\hline 17 & Austria & 7.5 & 0.8 & 9 \\
\hline 18 & Estados Unidos & 7.5 & 09 & 8 \\
\hline 19 & Israel & 7.1 & 1.4 & 9 \\
\hline 20 & Chile & 6.8 & 0.9 & 9 \\
\hline 21 & Francia & 6.7 & 0.6 & 9 \\
\hline 27 & Costa Rica & 5.6 & 1.6 & 5 \\
\hline 41 & Perú & 4.5 & 0.8 & 6 \\
\hline 42 & Uruguay & 4.3 & 0.9 & 3 \\
\hline 46 & Brasil & 4.0 & 0.4 & 9 \\
\hline 56 & México & 3.3 & 0.6 & 9 \\
\hline 61 & Argentina & 3.0 & 0.6 & 9 \\
\hline 69 & Bolivia & 2.8 & 1.2 & 4 \\
\hline 77 & Ecuador & 2.3 & 1.5 & 3 \\
\hline 78 & Venezuela & 2.3 & 0.8 & 9 \\
\hline 79 & Colombia & 2.2 & 0.8 & 9 \\
\hline 83 & Honduras & 1.7 & 0.5 & 3 \\
\hline 84 & Paraguay & 1.5 & 0.5 & 3 \\
\hline 85 & Camerún & 1.4 & 0.5 & 4 \\
\hline
\end{tabular}


La corrupción, al ser un fenómeno que afecta a toda la sociedad, involucra a toda su estructura fundamental: el tipo de democracia, la administración pública, los partidos políticos, la empresa, la educación, la familia, etc. (Pacheco, 1996, citado en Garda y otros, 1999) ${ }^{6} \mathrm{y}$, por tanto, produce diversos efectos en los aspectos morales, económicos, sociales y/o políticos de la sociedad.

Dentro de los efectos que puede producir la corrupción en el ámbito social y/o político, se encuentra un aumento de la desconfianza que posee la ciudadanía en lo referente a sus representantes, lo que afecta a las instituciones encargadas de impartir justicia, garantizar la seguridad ciudadana y el control de la administración pública. (Zuluaga, 1996 en Carda y otros, 1999) ${ }^{6}$. Debido a lo anterior, se produce un alejamiento entre el ciudadano y las instituciones; este alejamiento de la sociedad frente al Estado hace de la corrupción un proceso rápido ya que en la medida que se afianza se hace más difícil restablecer una relación de confianza (Pacheco, 1996, en Carda y otros, 1999) ${ }^{6}$.

Un efecto importante de la corrupción es el costo económico que genera en un país, expresada en la pérdida de recursos, lo que trae como consecuencia que los bienes públicos no se encuentren al servicio de los más necesitados. Junto a esto la acción de los funcionarios públicos corruptos puede hacer que la energía de los empresarios se desvíe desde las actividades productivas hacia la búsqueda de Renta. (Informe Comisión Nacional de Etica Pública, 1994) ${ }^{12}$. La corrupción está asociada a un mayor déficit fiscal con el consecuente impacto sobre las políticas de estabilización de un país. (Informe Comisión Nacional de Ética Pública, 1994) ${ }^{12}$

Por otro lado los costos de la corrupción también pueden subdividirse en términos tales como:

- Eficiencia: tiene que ver con el mal uso de recursos, desaprovecharlos, como lo sería crear males públicos y distorsionar las políticas de un país.

- Distribución: se distribuiría de mala manera los recursos públicos, siendo mayor la tajada de los ricos en desmedro de los pobres.

- Incentivos: trae como consecuencia la baja de energía de los funcionarios y ciudadanía y la tendencia hacia la búsqueda socialmente improductiva de rentas corruptas.

- Política: crea cinismo popular e inestabilidad en el régimen político. Como dato anecdótico, se plantea que la corrupción puede tener beneficios en algunos casos. Como ejemplo se puede citar el hecho que cuando la legislación vigente, no se ha basado en el «interés social» o sólo beneficia a selectos grupos, entonces la corrupción permite evitar una «mala legislación». (Aedo, 1995, citado en Carda y otros, 1999$)^{6}$

Como quiérase que el hombre es protagonista, es quien puede asumir las medidas correctivas o continuar envuelto en la corrupción, por ello pensamos que las personas asumen sus responsabilidades y éstas responden en gran medida a las atribuciones contenidas en las disposiciones psicológicas con las que cuenta, disposiciones que ha ido adquiriendo a la largo de la vida y van configurando patrones de comportamiento estables con las que el individuo se enfrenta ante diversas situaciones, eventos o circunstancias cuya forma típica queremos identificar en el presente estudio, averiguando si es el estilo atribucional optimista el que nos caracteriza o por lo contrario el pesimista ante la 
corrupción para lo cual cuando trabajamos con una muestra de púberes y adolescentes que cursan el primer grado hasta el quinto de secundaria y jóvenes universitarios de gestión pública despejar la siguiente interrogante: La edad, el sexo y el grado de instrucción plantea diferencias en las disposiciones psicológicas hacia la corrupción?

El presente estudio se propone identificar el estilo atribucional que tipifica a los púberes, adolescentes y jóvenes estudiantes. Así mismo queremos identificar el papel de la edad, el sexo y grado de instrucción en las dimensiones de: control externo o interno, estabilidad o inestabilidad, especificidad o globalidad e importancia que para el examinado tiene la posibilidad de enfrentarse ante una situación de corrupción. 


\section{MATERIALES Y MÉTODOS.}

Para despejar la interrogante planteada, nos hemos remitido a las datos de la oficina de estadística del ministerio de Educación cuya población de estudiantes de nivel secundaria a nivel de Lima Metropolitana es de 440,877 donde 217,929 (49\%) son varones y 222,948 (51\%) son mujeres. Utilizando las datos de la Asamblea Nacional de Rectares al aña 2002, en las universidades nacionales del departamento de Lima, albergan a un total de 811,268 estudiantes, de las cuales 48,166 (59\%) son varones y 33,102 (41\%) son mujeres. Para ambas estratos hemos estimado un tamaño muestral al 0.05 de margen de error con un nivel de confianza del 0.95 . Tomando en cuenta las totales de cada estrato a partir de la estimación de sus varianzas, requerimos 657 escolares distribuidas del primero al quinto de secundaria y 212 estudiantes universitarios distribuidas en las diferentes universidades de gestión estatal del departamento de Lima.

El método de investigación es descriptivo, diseño comparativa transversal, Hernández Sampieri (1997) ${ }^{13}$

Para medir las disposiciones psicológicas fue mediante la teoría del estilo atribucional mencionada líneas arriba, a partir de la cual se trabaja can la escala ASQCIDE, de Peterson y Seligman (1991) ${ }^{1}$ adaptada par Vicuña, L. (1999) ${ }^{2}$ a condiciones de desastre, y $\left(2001^{3}\right.$ a las condiciones de ingreso a la universidad; y esta vez hacia el acta corrupta, para la cual se consideró cama indicadores para la validez de contenido atropella a la libertad, justicia, obediencia, puntualidad, responsabilidad, honradez, intimidad, lealtad, cooperación, acceso a la información y a la seguridad; entendidas como deberes y derechas más que en su acepción coma valores en algunas de ellas.

Estas indicadores fueran adaptadas a las dimensiones de Internalidad -Externalidad, Estabilidad - Inestabilidad, Especificidad - Globalidad y también a la evaluación de la importancia del contenido del ítem.

La confiabilidad fue estimada con un grupa de 100 constituidas por púberes de adolescentes y jóvenes de ambas sexos por el método del test-retest con intervalo de 3 semanas entre la primera y la segunda aplicación, obteniendo un coeficiente de correlación de 0.92, y un índice de fiabilidad de 0.96. La validez fue para el método de análisis de contenido por el juicio de expertas seleccionando aquellas contenidas que de acuerdo a la opinión de 10 psicólogos con experiencia en la psicología del adolescente y joven dieran su respuesta favorable equivalente a un Ji cuadrada para el 0.01 de margen de error.

La aplicación fue de forma colectiva en grupas no mayares de 25 par vez, cuidando en todas las casas que las examinadas comprendieran el procedimiento para responder a la escala. Al respecta es conveniente señalar que el contenido funciona de manera limitada con las estudiantes del primero y segundo de secundaria cuya edades corresponde a las 12 y 13 años de edad respectivamente, la que deja entre ver que la comprensión del contenido del estila atribucional demanda del examinada pensamiento reversible con apoyo estrictamente verbal, esto es que pueda realizar juicios hipotéticos proposicionales donde la palabra sustituye a la concreta; por las datos obtenidos esta capacidad cognitiva aún no está consolidada mayoritariamente en estos segmentos cronológicos, pero si a partir de los 14 años de edad donde desaparece el afronte anecdótico del examinado para dar lugar a una hipotética situación vivenciada que es lo que demanda el test. 
La prueba está constituida por 12 situaciones donde cada una expone al sujeto a un posible acto de corrupción la que deberá resolver en cada una de las tres siguientes dimensiones: Externalidad Internalidad, Estabilidad - Inestabilidad, Especificidad Globalidad, además se incluyen dos ítems, uno para evaluar la causa fundamental a la que el examinado atribuye la situación planteada; y un ítem que evalúa la importancia que el sujeto otorga a esa situación; lo que hace que cada una de esas situaciones contenga 5 preguntas, conteniendo la escala un total 60 ítems cuyo tiempo promedio estimado de desarrollo es de 45 minutos en estudiantes de secundaria y 30 minutos en estudiantes universitarios.

Combinando las respuestas de las tres dimensiones, el diagnostico a la que se arriba es, Estilo Atribucional Optimista (Externalidad + Inestabilidad + Especificidad) y Pesimista (Internalidad + Estabilidad + Globalidad $)$ u otra combinación que no mencionada aquí.

\section{RESULTADOS}

El presente estudio quiere explorar en qué medida el grado escolar que lleva implícito la edad cronológica es una variable que afecta a las dimensiones del estilo atribucional hacia situaciones que plantean un acto corrupto; así mismo, queremos averiguar si el sexo plantea diferencias significativas para concluir estimando el porcentaje de jóvenes con estilo atribucional Optimista o Pesimista.

El análisis a efectuarse será para cada una de las dimensiones y los ítems que complementan a la escala mencionada, que son: la autopercepción de la causa y la importancia que el sujeto otorga a la situación. Empecemos analizando el papel que cumple la edad cronológica sobre las dimensiones:

En la dimensión Externalidad Internalidad, comparando las puntuaciones obtenidas por los examinados del primero al quinto de secundaria y estudiantes universitarios, hemos encontrado una razón $\mathrm{F}$ de 7.34 que resulta muy significativa aún para el 0.01 de margen de error, lo que indica que el grado escolar que lleva implícito la edad cronológica es una variable que diferencia las respuesta de Internalidad - Externalidad, tal como puede verse en la tabla 1.

Tabla 1.- Resumen de Análisis de varianza de la Dimensión Externalidad Internalidad en función al grado escolar.

\begin{tabular}{|l|c|c|c|c|c|c|}
\hline Fuente de Variación & $\mathrm{Ss}$ & $\mathrm{gl}$ & $\mathrm{S}_{\mathrm{e}}^{2}$ & $\mathrm{~S}_{\mathrm{D}}^{2}$ & $\mathrm{~F}$ & $\mathrm{~F}^{\prime} 0.05$ \\
\hline Entre Grupo & 35.4056 & 4 & 8.8514 & & & \\
Dentro Grupo & 1041.7754 & 864 & & 1.2058 & $7.3409 * * *$ & 2.3822 \\
Total & 1064.1810 & 868 & & & & \\
\hline
\end{tabular}

$* * * p<0.01$

Analizando las medias aritméticas de los grados escolares y de la universidad se observa que a menor edad los examinados se ubican en el grado quinto de una escala de ocho grados que corresponde a tendencia a la Internalidad respuesta que se modifica hacia tendencia a la externalidad a partir del tercer grado de educación secundaria; pero es 
preocupante que terminando el quinto año de secundaria incluyendo la universidad las medias aritméticas estén inclinando hacia el grado quinto, es decir hacia un retorno hacia la tendencia Internalidad, tal como puede verse en la tabla 2.

Tabla 2.- De Medias Aritméticas y Desviación Estándar de la Dimensión Externalidad - Internalidad por grados escolares.

\begin{tabular}{|c|c|c|c|}
\hline Grados & $\mathrm{n}$ & $\mathrm{X}$ & $\mathrm{DS}$ \\
\hline 1ro y & 72 & 5 & 1.09 \\
2do & 64 & 4013 & 0.83 \\
3ro & 206 & 4.78 & 1.20 \\
4to & 315 & 4.86 & 1.04 \\
5to & 212 & 4.9 & 1.14 \\
Univ. & & & \\
\hline
\end{tabular}

En la dimensión Inestabilidad Estabilidad, también encontramos diferencias significativas en función al grado académico que implica una edad cronológica determinada, la razón $\mathrm{F}$ encontrada es también muy significativa aún para el 0.01 de margen de error, lo que deja entrever que la edad cronológica y el grado escolar son variables asociadas a la dimensión Inestabilidad - Estabilidad del estilo atribucional, tal como puede verse en la tabla 3.

Tabla 3.- Resumen de Análisis de varianza de la Dimensión Estabilidad Inestabilidad en función al grado escolar.

\begin{tabular}{|l|c|c|c|c|c|c|}
\hline $\begin{array}{l}\text { Fuente de } \\
\text { Variación }\end{array}$ & $\mathrm{Ss}$ & $\mathrm{gl}$ & $\mathrm{S}^{2}$ & $\mathrm{~S}_{\mathrm{p}}^{2}$ & $\mathrm{~F}$ & $\mathrm{~F}^{\prime} 0.05$ \\
\hline $\begin{array}{l}\text { Entre Grupo } \\
\text { Dentro Grupo } \\
\text { Total }\end{array}$ & $\begin{array}{c}24.9757 \\
1074.9458\end{array}$ & 4 & 6.2439 & & & \\
1099.9214 & 868 & & 1.2442 & $5.0186^{* * * *}$ & 2.3822 \\
\hline
\end{tabular}

$* * * p<0.01$

Analizando el valor de las medias aritméticas encontramos que todos los grupos estudiados están comprendidos del grado cuatro hacia abajo, lo que ubica a los examinados en la categoría de tendencia a la inestabilidad, es decir asumen que, lo que ocurre es algo que no estará presente siempre. Cabe destacar que nuevamente es el tercer año de secundaria quienes se definen dentro del campo del grado tres, lo que indica mayor consideración que lo que ocurre no estará nunca más; en tanto que los estudiantes universitarios describen una media de 4.59 inclinándolos hacia el grado quinto, esto es 
hacia la tendencia a considerar que la situación de corrupción va a permanecer, tal como puede verse en la tabla 4.

Tabla 4.- De Medias Aritméticas y Desviación Estándar de la Dimensión Estabilidad Inestabilidad por grados escolares.

\begin{tabular}{|c|c|c|c|}
\hline Grados & $\mathrm{n}$ & $\mathrm{X}$ & $\mathrm{DS}$ \\
\hline 1ro y 2do & 72 & 4.25 & 1.22 \\
3ro & 64 & 3.98 & 0.65 \\
4to & 206 & 4.24 & 1.08 \\
5to & 315 & 4.4 & 1.17 \\
Univ. & 212 & 4.59 & 1.14 \\
\hline
\end{tabular}

En la dimensión Especificidad Globalidad hemos encontrado una razón F de 1.84 que no resulta significativa, lo que indica que los grados escolares y la edad cronológica es una variable no asociada para la ubicación hacia un grado determinado de respuesta, tal como puede verse en la tabla 5 .

Tabla 5.- Resumen de Análisis de varianza de la Dimensión Especificidad Globalidad en función al grado escolar.

\begin{tabular}{|c|c|c|c|c|c|c|}
\hline $\begin{array}{c}\text { Fuente de } \\
\text { Variación }\end{array}$ & Ss & $\mathrm{gl}$ & $\mathrm{S}^{2}$ & $\mathrm{~S}_{\mathrm{p}}^{2}$ & $\mathrm{~F}$ & $\mathrm{~F}^{\prime} 0.05$ \\
\hline $\begin{array}{c}\text { Entre Grupo } \\
\text { Dentro Grupo } \\
\text { Total }\end{array}$ & $\begin{array}{c}1129.6434 \\
1138.8956\end{array}$ & 4 & 2.4108 & 1.3070 & 1.8446 & \\
\hline
\end{tabular}

$* * * p<0.01$

Para identificar el grado de localización de las respuestas revisamos las medias aritméticas, encontrando que estas sin mayor variabilidad se encuentran en el grado cuatro lo que indica tendencia hacia la Especificidad, esto es atribuir que la situación de corrupción vivenciada solo influye en esa situación concreta, tal como puede verse en la tabla 6 . 


\section{Tabla 6.- De Medias Aritméticas y Desviación Estándar de la Dimensión Especificidad - Globalidad por grados escolares.}

\begin{tabular}{|c|c|c|c|}
\hline Grados & $\mathrm{n}$ & $\mathrm{X}$ & $\mathrm{DS}$ \\
\hline 1ro y 2do & 72 & 4.09 & 1.12 \\
3ro & 64 & 4.38 & 0.54 \\
4to & 206 & 4.22 & 1.18 \\
5to & 315 & 4.41 & 1.12 \\
Univ. & 212 & 4.37 & 1.27 \\
\hline
\end{tabular}

La importancia que los examinados otorgan a la situación de corrupción plantada según el grado escolar y la edad cronológica presentan una razón F de 2.79 significativa al 0.05 . Ver tabla 7.

Tabla 7.- Resumen de Análisis de varianza de la Dimensión Importancia en función al grado escolar.

\begin{tabular}{|c|c|c|c|c|c|c|}
\hline $\begin{array}{l}\text { Fuente de } \\
\text { Variación }\end{array}$ & Ss & gl & $S^{2}$ & $S_{p}^{2}$ & $\mathrm{~F}$ & $F^{\prime} 0.05$ \\
\hline Entre Grupo & 16.1416 & 4 & 4.0354 & & $1.2 .7986^{* *}$ & \\
\hline Dentro Grupo & 1245.8321 & 864 & & 1.4419 & & 2.3822 \\
\hline Total & 1261.9737 & 868 & & & & \\
\hline
\end{tabular}

$* * * p<0.01$

Analizando los valores de la media aritmética encontramos que los estudiantes de menor grado escolar, tercero de secundaria hacia bajo se ubican en el grado cinco, lo que interpretamos como tendencia a otorgarle importancia a la situación vivenciada, pero a partir del quinto de secundaria hacia delante la ubicación promedio es en el grado 6 lo que indica que a mayor grado de instrucción y edad se otorgan más importancia a las situaciones donde el sujeto está en situación vivenciada de un acto de corrupción, como se puede observar en la tabla 8. 


\section{Tabla 8.- De Medias Aritméticas y Desviación Estándar de la Dimensión Importancia por grados escolares.}

\begin{tabular}{|c|c|c|c|}
\hline Grados & $\mathrm{n}$ & $\mathrm{X}$ & $\mathrm{DS}$ \\
\hline 1ro y 2do & 72 & 5.59 & 1.41 \\
3ro & 64 & 5.67 & 0.76 \\
4to & 206 & 5.82 & 1.21 \\
5to & 315 & 6.02 & 1.15 \\
Univ. & 212 & 5.94 & 1.28 \\
\hline
\end{tabular}

Veamos ahora los resultados cuando contrastamos las dimensiones del estilo atribucional en función al sexo.

La dimensión Externalidad - Internalidad y el sexo.

En la muestra total encontramos medias aritméticas correspondientes al grado cuatro pero con tendencia hacia el grado quinto, lo que deja entrever que tanto hombres como mujeres tienden hacia la Internalidad, cuya diferencia de medias aritméticas no es significativa. Tal como puede verse en la tabla 9.

Al analizar la Inestabilidad Estabilidad según el sexo encontramos que las medias aritméticas tanto de los hombres como las mujeres se ubican en el grado cuatro claramente definida correspondiendo a la tendencia hacia la Inestabilidad, sin encontrar diferencias significativas en función al sexo, esto es que el grupo examinado considera que tal acontecimiento nunca más estará presente. Ver tabla 9.

En la dimensión Especificidad Globalidad; encontramos que la media aritmética es definida en hombres y mujeres en el grado cuatro, sin diferenciarse significativamente, este promedio significa que ambos grupos consideran que la situación de corrupción vivenciada solo influyen en tal situación concreta y no en otras, tal como puede verse en la tabla 9.

Respecto a la importancia que otorgan los examinados a las situaciones vivenciadas, encontramos en los varones un promedio de 5.71 yen las mujeres de 6.04 , donde la diferencia es significativa aún para el 0.01 de margen de error, siendo las mujeres quienes al ubicarse en el grado seis comunican que le dan más importancia a la situación de corrupción vivenciada que los varones. Ver tabla 9.

En cuanto a la Causa Autopercibida las diferentes respuestas fueron agrupadas por su contenido en dos grandes grupos: aquello que decían que la causa de alguna manera y por alguna razón se debía a ellos frente a las respuestas de aquellos cuyos contenidos de causalidad eran adjudicados a terceros o a otras condiciones, para el primer caso se valoró con uno y en él se valoró con dos, por tanto el promedio aritmético estaría definiendo la proporcionalidad de sujetos que obtuvieron uno o que obtuvieron dos, donde una media aritmética de 1.5 significaría que la mitad de los examinados considera que la causa es interna y la otra mitad que la causa es externa. En el presente estudio hemos encontrado una media de 1.37 en hombre y una media de 1.36 en mujeres, que corresponde al $68 \%$ en ambos grupos, que atribuyen que la causa del contenido de la situación corrupta es interna, este $68 . \%$ tiene una contraparte del $32 \%$ cuya diferencia mediante el ji cuadrado es de 12.96 que para un grado de libertad el ji teórico es de 3.84, por tanto el porcentaje de 
sujetos que atribuyen que la causa es interna es significativa al 0.05 de margen de error, dato que no es favorable desde la teoría de la atribución porque es un elemento del pesimismo. Ver tabla 9.

Tabla 9.- De diferencias de Medias Aritméticas de la Causa Autopercibida, Dimensiones e Importancia Atribucional según el sexo.

\begin{tabular}{|c|c|c|c|c|c|c|c|c|c|c|}
\hline & \multicolumn{2}{|c|}{$\begin{array}{c}\text { Causa } \\
\text { Autopercibida }\end{array}$} & \multicolumn{2}{|c|}{$\begin{array}{l}\text { External- } \\
\text { Internal }\end{array}$} & \multicolumn{2}{|c|}{ Inestab-Estabi } & \multicolumn{2}{|c|}{ Especif-Global } & \multicolumn{2}{|c|}{ Importancia } \\
\hline & $\mathbf{H}$ & $\mathbf{M}$ & $\mathbf{H}$ & $\mathbf{M}$ & $\mathbf{H}$ & $\mathbf{M}$ & $\mathbf{H}$ & $\mathbf{M}$ & $\mathbf{H}$ & M \\
\hline $\mathbf{n}$ & 388 & 4.81 & 388 & 4.81 & 388 & 4.81 & 388 & 4.81 & 388 & 4.81 \\
\hline $\mathbf{X}$ & 1.37 & 1.36 & 4.84 & 4.79 & 4.34 & 4.39 & 4.34 & 4.32 & 5.71 & 6.04 \\
\hline DS & 0.22 & 0.23 & 1.07 & 1.15 & 1.11 & 1.14 & 1.10 & 1.18 & 1.22 & 1.18 \\
\hline $\mathbf{D S}^{2}$ & 1.05 & 0.05 & 1.14 & 1.32 & 1.24 & 1.29 & 1.22 & 1.39 & 1.47 & 1.38 \\
\hline Dif & \multicolumn{2}{|c|}{0.01} & \multicolumn{2}{|c|}{0.05} & \multicolumn{2}{|c|}{0.05} & \multicolumn{2}{|c|}{0.02} & \multicolumn{2}{|c|}{0.33} \\
\hline E.E. & \multicolumn{2}{|c|}{0.05} & \multicolumn{2}{|l|}{0.07} & \multicolumn{2}{|c|}{0.08} & \multicolumn{2}{|c|}{0.08} & \multicolumn{2}{|c|}{0.08} \\
\hline $\mathbf{t}$ & \multicolumn{2}{|c|}{0.19} & \multicolumn{2}{|c|}{0.66} & & & \multicolumn{2}{|c|}{0.28} & \multicolumn{2}{|c|}{$4.04 * *$} \\
\hline
\end{tabular}

$* * \mathrm{p}<0.01$

En cuanto al diagnóstico de los estilos atribucionales Optimista y Pesimista, hemos encontrado en la distribución de frecuencias absolutas y relativas para el estilo atribucional optimista y pesimista, agrupados según el sexo y ordenados por el grado de instrucción y edad cronológica, que el estilo atribucional predominante es el optimista, tanto en los hombres como en las mujeres, con distribuciones porcentuales muy similares, dejando entrever que en la muestra general, tanto los adolescentes como lo jóvenes en el continuo cronológico e instruccional se caracterizan por el predominio de la Externalidad, asociado con la inestabilidad de los acontecimientos y la especificidad de los hechos en un 73 por ciento en tanto que el 27 por ciento, presenta estilo atribucional Pesimista es decir con predominio de la Internalidad, la estabilidad de los acontecimientos y la globalidad de los mismos, con un valor ji cuadrado de 21.16 que resulta muy significativo aun para el 0.01 de margen de error, tal como puede verse en la tabla 10.

Con el propósito de describir con mayor detalle hemos agrupado a los examinados por el grado de instrucción y el género, para calcular sus frecuencias absolutas y relativas según el diagnóstico de estilo atribucional optimista y pesimista, en función al género, nivel instruccional, y para verificar si los porcentajes son significativos en cada categoría analizada hemos calculado el ji cuadrado encontrando:

En la muestra de escolares el 76 por ciento presentan un estilo atribucional optimista, con un ligero predominio en los varones, el cálculo del ji cuadrado indica que lo hallado es muy significativo aún para eI 0.01 de margen de error. 
Tabla 10.- De Frecuencias absolutas y porcentuales respecto a los estilos atribucionales Optimista y Pesimista hacia los actos de corrupción agrupados según el sexo y grado de instrucción.

\begin{tabular}{|c|c|c|c|c|c|c|c|c|c|c|c|c|}
\cline { 2 - 13 } \multicolumn{1}{c|}{} & \multicolumn{4}{c|}{ OPTIMISMO } & \multicolumn{5}{c|}{ PESIMISMO } \\
\cline { 2 - 13 } & Hombres & Mujeres & \multicolumn{2}{c|}{ Total } & \multicolumn{2}{c|}{ Hombres } & \multicolumn{2}{c|}{ Mujeres } & \multicolumn{2}{c|}{ Total } \\
\cline { 2 - 13 } & F & $\%$ & F & $\%$ & F & $\%$ & F & $\%$ & F & $\%$ & F & $\%$ \\
\hline 1ro y 2do & 31 & 4 & 24 & 3 & 55 & 6 & 10 & 1 & 7 & 1 & 17 & 2 \\
3ro & 25 & 3 & 37 & 4 & 62 & 7 & 0 & 0 & 2 & 0 & 2 & 0 \\
4to & 81 & 9 & 79 & 9 & 160 & 18 & 20 & 2 & 26 & 3 & 46 & 5 \\
5to & 84 & 20 & 138 & 16 & 222 & 26 & 33 & 4 & 60 & 7 & 93 & 11 \\
Univ. & 65 & 7 & 74 & 4 & 139 & 16 & 39 & 4 & 34 & 4 & 73 & 8 \\
Sub.total & 286 & 33 & 352 & 41 & 638 & 73 & 102 & 12 & 129 & 15 & 231 & 27 \\
\hline
\end{tabular}

En la muestra de universitarios el 66 por ciento presenta estilo atribucional optimista, donde en los universitarios el ji cuadrado es solo significativo al 0.05 de margen de error.

En lo totales el ji cuadrado resulta muy significativo al 0.01 de margen de error, lo que indica que mínimamente el 73 por ciento de adolescentes y jóvenes presentan estilo atribucional optimista hacia las situaciones de corrupción, estos resultados puede verse en la tabla 10.

Tabla 11.- De frecuencias absolutas y relativas y cálculo del Ji cuadrado correspondiente al estilo atribucional optimista pesimista

\begin{tabular}{|c|c|c|c|c|c|c|c|}
\hline & \multicolumn{3}{|c|}{ Optimismo } & \multicolumn{4}{|c|}{ Pesimismo } \\
\hline & $\mathrm{n}$ & $\mathrm{F}$ & $\%$ & $\mathrm{~F}$ & $\%$ & $X^{2}$ & \\
\hline Escolares Varones & 288 & 221 & 77 & 63 & 22 & 29 & $* *$ \\
\hline Escolares Mujeres & 373 & 278 & 75 & 95 & 25 & 24 & $* *$ \\
\hline TOT AL DE ESCOLARES & 657 & 499 & 76 & 158 & 24 & 27 & $* *$ \\
\hline Universitarios & 104 & 65 & 63 & 39 & 38 & 6.3 & $*$ \\
\hline Universitarias & 108 & 74 & 69 & 34 & 31 & 14 & $* *$ \\
\hline TOTAL DE UNIVERSITARIOS & 212 & 139 & 66 & 73 & 34 & 9.7 & $* *$ \\
\hline Varones en Total & 388 & 286 & 74 & 102 & 26 & 22 & $* *$ \\
\hline Mujeres En Total & 481 & 352 & 73 & 129 & 27 & 21 & $* *$ \\
\hline GRUPO TOTAL & 869 & 638 & 73 & 231 & 27 & 22 & $* *$ \\
\hline
\end{tabular}

$* P<0.05 P<0.01$ 


\section{DISCUSIÓN}

Cuando recogíamos información algunos niños del cuarto y quinto grado, cuyas edades fluctúan entre los 10 Y 11 años de edad, preguntaban con curiosidad que se venia haciendo, cuando les contestábamos, mostraban interés y sabiéndonos psicólogos, nos decían «psicólogo, yo doy la prueba» y/ su interés era tanto que decidimos aplicarles, en curso a ello, pronto descubrimos que les costaba esfuerzo ponerse en situaciones hipotéticas, por ejemplo decían «como contesto, si a mí no me falta el dinero», se les aclaraba diciéndoles, haz de cuenta, supón que te hace falta el dinero, replicaban «pero como si no me falta», y de esa manera reaccionaban con casi todas las situaciones planteadas en los diversos ítemes. Por estas reacciones fácilmente se visual iza que en este rango de edad, si bien el pensamiento es lógico reversible, Piaget J, (1969) ${ }^{4}$, requieren de apoyo concreto, aun no pueden utilizar solo el lenguaje para desarrollar operaciones hipotéticas, de esta manera sus respuestas resultan anecdóticas, por ejemplo «Ah cuando mi papá no tenia plata estaba de mal humor" y otras respuestas similares.

A partir de los 12 y 13 años primero y segundo de secundaria los púberes ya son capaces de hipotetizar, solo encontramos dificultad en un diez por ciento de la muestra de estos grupos, viéndonos obligados a no incluirlos en el estudio. Cuando examinamos s estratos de mayor edad, a partir del tercer grado hasta el quinto de secundaria y con estudiantes universitarios, no encontramos ninguna dificultad. En todos los casos su respuestas fluían con facilidad en la medida que leían los diferentes ítemes.

Como ya describimos la disposición psicológica hacia la corrupción ha sido asumida como el estilo explicativo optimista o pesimista en este caso hacia la corrupción, es decir a que atribuyen que las personas de todas las condiciones sociales actúen ante la corrupción como si esta se encontrará generalizada a tal punto de convivir con ella, conoce si el estilo atribucional es optimista sería una fortaleza de nuestra juventud que debería movilizarse hacia su fortalecimiento, pero si fuera de un estilo pesimista, sería una gran debilidad con un panorama incierto y cada vez una sociedad más anómala y caótica, altamente personalizada y centrada en sus propios benéficos a costa de cualquiera y de cualquier precio. Para analizar este estado tuvimos prilllero que explorar las dimensiones que Peterson y Seligman ${ }^{1}$ consideran como elementos del estilo atribucional, es decir la dimensión Externalidad - Internalidad, es decir si la persona asume que una situación dada se debe a otras causas o circunstancias o si por lo contrario se debe a él mismo. La otra dimensión es la Estabilidad -Inestabilidad, se refiere la estabilidad a suponer que la causa de un determinado acontecimiento siempre estará presente, la Inestabilidad se refiere a lo contrario que la causa del acontecimiento no estará presente nunca más. La tercer dimensión es la Especificidad - Globalidad, en esta dimensión se asume la causa que determino un determinado acontecimiento influye solo en esa situación concreta, la Globalidad, por lo contrario asume que la causa influirá en todas las situaciones de la vida.

Combinando estas dimensiones obtenemos el estilo explicativo, cuando la combinación es: Externalidad, Inestabilidad, Especificidad, la persona tiene un estilo explicativo Optimista si la combinación es otra como esta: Internalidad, Estabilidad, Globalidad, el estilo explicativo es Pesimista. 
De los resultados se desprende que el sesenta y ocho por ciento de la muestra examinada considera que la causa de un determinado acontecimiento se debe a ellos mismos, escribiendo respuesta como estas, "porque yo provoco envidia» o "Gasté más dinero de lo que tenía» y respuestas parecidas don el eje causal sale de uno mismo, el 'treinta y dos por ciento, de los examinados mencionan que la causa está fuera de ello, cuando comunican "Las personas tendrán sus propios problemas» o "Las circunstancias como los amigos pueden desfinanciarnos». Por este dato aisladamente podríamos 'pensar que nuestro jóvenes se caracterizan por la Internalidad, sin embargo cuando exploramos los datos de la dimensión Externalidad- Internalidad, encontramos que los adolescentes que cursan el primero y segundo de secundaria cuyas edades fluctúan entre los 12 y 13 años tienden efectivamente hacia la Internalidad, pero se inicia un descenso hacia la tendencia a la Externalidad a partir del tercer grado, es decir hacia los 14 años de edad, sin embargo en los estudiantes universitarios nuevamente vemos un sesgo hacia la Internalidad, por estos datos nuestra juventud se encuentra en un punto crítico esto es el 68 por ciento se orienta aunque no de forma definitiva hacia la Internalidad que implica sentimiento de culpabilidad con los efectos negativos que este sentimiento conlleva, el 32 por ciento se encuentra hacia la Externalidad, esto es con un Yo más fuerte para enfrentar las diferentes presiones a las que la vida misma nos expone.

Analizando la dimensión Inestabilidad - Estabilidad, encontramos que la edad cronológica plantea diferencias, donde los estudiantes que cursan el tercero de secundaria y cuya edad cronológica corresponde a los 14 años, se ubican en tendencia a la Inestabilidad; en tanto que edades menores y mayores se ubican en tendencia a la Estabilidad. Este dato nos dice que el común de nuestros adolescentes y jóvenes consideran que la causa que explica un acto corrupto estará siempre presente, esto indica que nuestros jóvenes se inclinan hacia la permanencia de los acontecimientos, que en este punto no es saludable, ya que es un componente del estilo explicativo pesimista, lo hallado es común en los varones y en las mujeres.

Respecto a la dimensión Especificidad - Globalidad, no hemos encontrado diferencias significativas según la edad, las puntuaciones promedio están alrededor del grado cuatro que indica tendencia hacia la Especificidad, manteniéndose sin variación significativa en los estudiantes universitarios, quedando establecida la tendencia a la Especificidad, esto es, la atribución que la causa que lleva a un acto corrupto va a influir sólo en ciertas situaciones concretas de la vida. En esta dimensión el sexo tampoco plantea diferencias, dejando entre ver que tanto los varones como las mujeres comparten esta característica atribucional.

En cuanto a la Importancia que tienen los actos corruptos para la muestra examinada, plantea diferencias significativas las que van aumentado en la media que aumenta la edad cronológica, llegando los estudiantes de quinto grado de secundaria, y estudiantes universitarios a ubicarse en el grado seis de una escala de ocho grados. En este punto la edad el sexo si plantea las diferencias significativas siendo las mujeres quienes otorgan significativamente más importancia a los actos corruptos, cuyo promedio se ubica en el grado seis en tanto que los varones en el grado cinco. 
Combinando las dimensiones de Externalidad - Internalidad, Inestabilidad Estabilidad, Especificidad - Globalidad como ya señaláramos líneas arriba define el estilo explicativo; en el presente estudio de conformidad con las medias aritméticas encontradas el promedio en la dimensión Externalidad - Internalidad es de 4.82, que corresponde a una tendencia marcada a la Internalidad; en la dimensión Inestabilidad.

- Estabilidad la media aritmética es de 4.36, que ubica al grupo estudiado en el grado cuatro con tendencia a la Inestabilidad; y en la dimensión Especificidad - Globalidad la media aritmética es 4.35, ubicando al grupo examinado con tendencia a la Especificidad. De esta combinación el estilo explicativo se orienta hacia una tendencia al optimismo; debiendo reparar que la dimensión Internalidad es el componente que en nuestra juventud debería modificarse porque es generador de sentimientos de culpa que puede arrastrar a estados de melancolía hasta la depresión. Este dato llevado a porcentajes nos dice el $73 \%$ de nuestros examinados tienen características de un estilo explicativo optimistas con un $27 \%$ de jóvenes con estilo explicativo pesimista.

Este resultado no es del todo bueno, puesto que más de un cuarto de púberes y. jóvenes consideran que los actos corruptos se deben a ellos mismos, que siempre estará presente la situación corrupta y que tal acontecimiento acompañará a todas las situaciones de su vida. 


\section{CONCLUSIONES}

1. La edad cronológica plantea diferencias significativas en la dimensión Externalidad Internalidad, donde a menor edad es mayor la Externalidad.

2. En la dimensión Estabilidad Inestabilidad, la edad cronológica plantea diferencias significativas donde a menor edad hay mayor Inestabilidad.

3. En la dimensión Especificidad Globalidad la edad cronológica no plantea diferencias significativas, el grupo en su conjunto tiende hacia la Especificidad.

4. La importancia que cobran los actos corruptos son altamente significativas en la medida que aumenta la edad.

5. El sexo no plantea diferencias en las dimensiones Externalidad - Internalidad, Estabilidad -Inestabilidad, Especificidad Globalidad; solo hemos encontrado diferencia en la importancia que se concede a los actos corruptos, siendo las mujeres quienes otorgan más importancia que los varones.

6. La tendencia del grupo examinado hasta un $73 \%$ corresponde al estilo explicativo optimista, la diferencia presentan estilo explicativo pesimista.

7. Los púberes adolescentes y jóvenes atribuyen que los actos corruptos son susceptibles de ser manejados por ellos mismos, hecho que es alentador. 


\section{REFERENCIAS}

1. Peterson,c. The Meaning an Measurement of Explanatory Style, In. Psychological lnquiry. Vol.2 $\mathrm{N}^{\circ} 1,1-10.1991$.

2. Vicuña, L. Disposiciones psicológicas ante los diferentes tipos de afronte a estados de emergencia de origen natural y social. Revista de Investigación en Psicología Vol. 2 № 2. Lima Perú. 1999.

3. Vicuña, L. Concordancia entre la facultad académica a la que pertenece el alumno con los interés vocacionales y con los estilos atribucionales de las causas que determinaron el ingreso a la U.N.M.S.M en el concurso de admisión del 2000. Revista de Investigación en Psicología Vol. 4 N 1. Lima - Perú. 2001.

4. Weiner, B. An attributional theory of motivation and emotion. New York: SpringerVerlang. 1986.

5. M, Seligman Helplessness. On depression, development and death. San Francisco. B.H Freeman. 1975.

6. Carcía, M., Hormazábal, N., Mosalve, D., Rayo, C., Riffo, N., Rojas, T. Y Rosales, X El fenómeno de la corrupción desde una perspectiva psicosocial. Seminario para optar al grado de licenciado en psicología. Concepción. 1999.

7. Silva. C. y Hernandez, M. Las formas cotidianas de la corrupción: un análisis de discurso. Revista latinoamericana de psicología.29.243-260, 1995.

8. Fernandez-Dolz, J.M, Norma perversa: hipótesis teóricas. Psicothema,5,91-101, 1993.

9. Huerta, R. Corrupción entre la sociedad y el poder. Visión,1-15 de agosto. 1992.

10. Huntintong, S. y Scott, S. Corrupción: policía y sociedad. Uam-Azcapotzalco. 1994

11. Transparency International.Indice de percepción de corrupción.1999.

12. Informe de Comisión Nacional de Ética Publica: Probidad, transparencia y responsabilidad al servicio de los ciudadanos. Santiago de Chile. 1994.

13. Hernandez, R. ;Fernandez, C.; Baptista, P. Metodología de la Investigación. México, McGRAW-HILL. 1997.

14. Piaget, J. and Inhelder, B. The Psychology of the child. New York, NY: Basic Books.1969. 